VOL. $4(1971), 155-158$.

\title{
A homomorphism theorem for projective planes
}

\author{
Don Row
}

We prove that a non-degenerate homomorphic image of a projective plane is determined to within isomorphism by the inverse image of any one point. An application gives conditions for the preservation of central collineations by a homomorphism.

Except for papers by D.R. Hughes [3] and L.A. Skornjakov [7], a growing list of examples $[4,5,6]$, and some non-existence results $[1,2$, 3], little general information concerning homomorphisms of projective planes is available. The aim of this note is to give a standard fundamental isomorphism theorem for these homomorphisms, and from it a simple coordinate-free derivation of Hughes' conditions [3; Theorems 4.1, $4.2,4.3]$ for the preservation of central collineations by a homomorphism.

ISOMORPHISM THEOREM. A non-degenerate homomorphic image of a projective plane is determined to within isomorphism by the inverse image of any one point.

Proof. Consider homomorphisms $h_{i}: \pi \rightarrow \pi_{i}$ of a plane $\pi$ into planes $\pi_{i}, i=1,2$. Let $A, B, \Gamma, \ldots$ be the points and $\alpha, \beta, \gamma, \ldots$ the lines of $\pi$. For convenience we identify each line with the set of points incident with it. Denoting the inverse image, or coset, with respect to $h_{i}$, containing any element $B$ by $[B]_{i}$ we assume $[A]_{1}=[A]_{2}$ for some point $A \in \pi$ (in which case we write [A] for both $[A]_{i}$.

Received 23 September 1970. This work was supported by National Research Council of Canada grant no. A-7251. 
We now prove $[B]_{1}=[B]_{2}$ for any $B \&[A]$, remembering that $[B]_{i}=U_{B}\left([B]_{i} \cap B\right)$, the union being taken over all $B \supset B$.

Each line of $h_{i} \pi$ contains at least three points and we select $\Gamma \in A B$ so that $\Gamma \notin[A], \Gamma \notin[B]_{1}$. Thus for any $\Lambda \in B \cap[B]_{1}$, $h_{1} \Lambda \Gamma=h_{1} A B$ ensuring that $\Lambda \Gamma \cap[A]$ is non-empty and $h_{2} \Gamma \Lambda=h_{2} A h_{2} \Gamma=h_{2} A B$. If $B \cap[A]$ is empty then $h_{2} A B \neq h_{2} B$, giving $h_{2} \Lambda=h_{2} \Lambda \Gamma \cdot h_{2} B=h_{2} \mathrm{~B}$ (even if $h_{2} \Gamma=h_{2} \mathrm{~B}$ ).

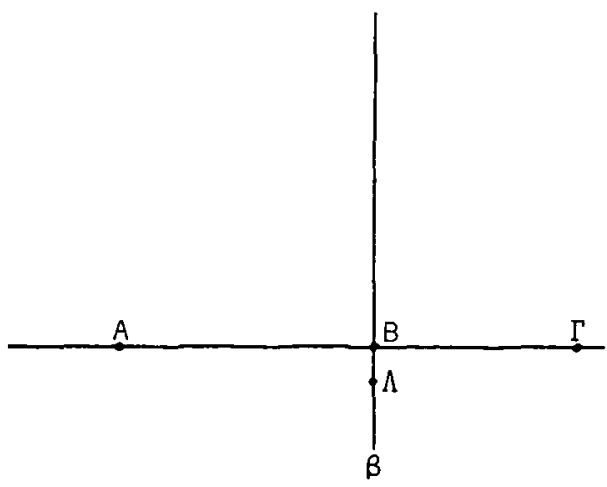

On the other hand, if $B \cap[A]$ is non-empty we first choose a point $\Delta$ satisfying $h_{1} \Delta h_{1} B, h_{2} \Delta h_{2} B$ as follows: select $\delta \supset B$ with $\delta \cap[A]$ empty and $\Delta \in \delta$ so that $\Delta \notin[B]_{2}$, and consequently by the above argument, $\Delta \nmid[B]_{1}$. There is a line $B^{\prime} \supset B$ whose image under $h_{1}$ does not contain either $h_{1} A$ or $h_{1} \Delta$. Thus any $\Lambda \in \beta \cap[B]_{1}$ is perspective from $\Delta$ to some point of $B^{\prime} \cap[B]_{1}$. Again by the preceding argument, $B^{\prime} \cap[B]_{1} \subseteq B^{\prime} \cap[B]_{2}$ and thus $\Lambda \in B \cap[B]_{2}$ (even if $\left.h_{2} \Delta \subset h_{2} B^{\prime}\right)$.

Hence $[\mathrm{B}]_{1} \subseteq[\mathrm{B}]_{2}$ and, interchanging the roles of $h_{1}$ and $h_{2}$, $[B]_{1}=[B]_{2}$. Thus the two homomorphisms have identical point cosets and, as $[A B]_{1}=\left\{A^{\prime} B^{\prime} \mid A^{\prime} \in[A], B^{\prime} \in[B]\right\}=[A B]_{2}$ whenever $[A] \neq[B]$, identical line cosets. Consequently the map $h_{1} \lambda \rightarrow h_{2} \lambda, h_{1} \Lambda \rightarrow h_{2} \Lambda$; $\lambda, \Lambda \in \pi$ is well defined and an isomorphism, $h_{1} \pi \rightarrow h_{2} \pi$. //

By considering $h_{1}$ and defining $h_{2}$ by $[A]_{2}=[A]_{1}$, $[B]_{2}=\left\{\Lambda \mid \Lambda \in \pi, \Lambda \notin[A]_{1}\right\},[A B]_{2}=\{\lambda \mid \lambda \in \pi\}$ for any two points $A, B$ in distinct cosets of $h_{1}$ we see that the theorem fails if one of the $h_{i} \pi$ is degenerate. 
THEOREM (Hughes). A hamomorphism having a non-degenerate image preserves a central collineation $g$ if and only if there are points $\mathrm{B}, g^{\mathrm{B}}$ having images distinct from the image of the centre, and not incident with the image of the axis, of the collineation.

Proof. Consider $h: \pi \rightarrow \pi^{\prime}$, and write $h_{1}=h, h_{2}=h g$. To show $g$ is preserved it suffices to show $[A]_{1}=[A]_{2}$ for any $A \in Y$ satisfying $h \mathrm{~A} \not \mathrm{B} \Gamma$ where $\Gamma$ is the centre, and $\gamma$ the axis, of $g$.

If $\Lambda \in[\mathrm{A}]_{l}$, writing $A^{\prime}=\Lambda \mathrm{B} \cdot \gamma$, we have $h \mathrm{~A}^{\prime}=h \mathrm{~A}$ and consequently $h g \Lambda=h \Gamma \Lambda \cdot h\left(\mathrm{~A}^{\prime} g \mathrm{~B}\right)=h \Gamma \Lambda \cdot h(\Lambda g \mathrm{~B})=h \mathrm{~A}=h g \mathrm{~A}$, that is $\Lambda \in[\mathrm{A}]_{2}$. As $h \mathrm{~A} \notin h \mathrm{~B} \Gamma \Longleftrightarrow h g \mathrm{~A} \notin h g \mathrm{~B} \Gamma$, we similarly consider $g^{-1}$ and show $[A]_{1}=[A]_{2}$.

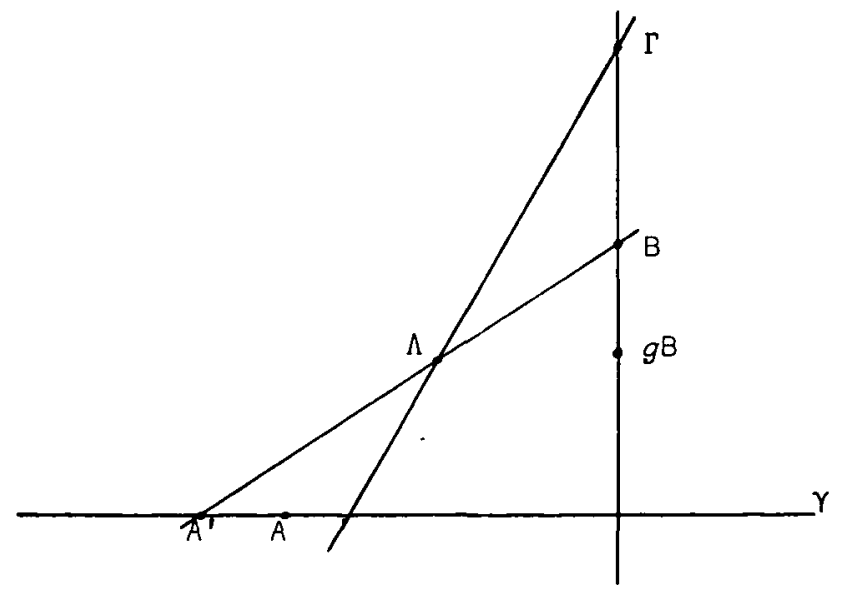

The converse is apparent. //

\section{References}

[1] Johannes André, "über Homomorphismen projektiver Ebenen", Abh. Math. Sem. Univ. Hamburg 34 (1969), 98-114.

[2] Peter Dembowski, "Homomorphismen von $\lambda$-Ebenen", Arch. Math. 10 (1959), 46-50.

[3] D.R. Hughes, "On homomorphisms of projective planes", Proc. Sympos. App 2. Math. 10, 45-52, (Amer. Math. Soc., Providence, Rhode Island, 1960). 
[4] Wilhelm Klingenberg, "Projektive Geometrien mit Homomorphismus", Math. Ann. 132 (1956), 180-200.

[5] Günter Pickert, Projektive Ebenen (Die Grundlehren der mathematischen Wissenschaften, Band 80 , Springer-Verlag, Berlin, Göttingen, Heidelberg, 1955).

[6] Don Row, "Sharply transitive projective planes and their homomorphisms", (Tech. Report Math. Dept., University of Tasmania, 15, 1967).

[7] L.A. Skornyakov, "Homomorphisms of projective planes and T-homomorphisms of ternary rings" (Russian), Mat. Sb. N.S. 43 (85) (1957), 285-294.

University of Tasmania,

Hobart, Tasmania. 